\title{
Research on Fault Location Method for Wind Turbine Line in Power System
}

\author{
LIU Yue ${ }^{1}$,GUAN Hong-mei ${ }^{2}$ \\ \{tosn614@163.com', dawda985@163.com²\} \\ (1. Department of Automation, College of Humanities Information Changchun University of \\ Technology, Changchun 130122,China; \\ 2.Department of Automation, College of Humanities Information Changchun University of \\ Technology,Changchun 130122,China)
}

\begin{abstract}
In the conventional fault location method of the wind turbine line, when multiple points are positioned in parallel, a location fault or a location error often occurs. Therefore, a fault location method for power system wind turbine is proposed. Identify the fault range of the power system wind turbine line, match the fault data within the controllable range, and correct the matching data to deviate from the execution range. The fault location of the wind turbine line of the power system is realized through the transformation. The simulation experiment is designed. The results show that the design method can achieve accurate positioning under multi-point fault location, and solve the problems in traditional methods.
\end{abstract}

Keywords: Power system; Wind turbine; Line fault; Fault location.

\section{Introduction}

Wind turbines are important components in the power system. The composition of the wind turbines is complex, and often in complex and changeable operating conditions. As a result, the wind turbine's line is one of the components with the highest failure rate, and its failure causes the longest downtime. Therefore, it is of great research interest to realize accurate and timely fault location of this component.[1-2]

When the extreme points of the signal from the wind turbine are not evenly distributed, the line resolver will cause "overshoot" and "undershoot" phenomena, resulting in aliasing in the power system. Starting from solving the problem of distribution of line fault points, by adding Gaussian white noise to the wind turbine to make the distribution of line fault points uniform, in order to reduce the impact of modal aliasing[3]. Human experience intervention, greatly reducing the adaptability of line fault location methods. Based on this, many researchers have proposed a variety of different methods for parameter tuning. To a certain extent, the blindness of artificial selection of parameters is avoided, but the selection of noise intervals in the fault location method still needs to be set manually, and there is a long time for pre-processing to select parameters.

The magnitude of line fault location deviation has a significant impact on the effect of 
wind turbines. When the noise is too small, the line fault location cannot solve the problem of power system aliasing. For this reason, a fault location method for power system wind turbine lines is proposed.

\section{Fault location method for power system wind turbine}

\subsection{Identify the fault range of the power system wind turbine line}

According to the characteristics of the power system, the search procedure of the wind turbine is set using CS technology to determine the effective range of the line fault location. Specify the line fault judgment step factor. This factor is the parameter for judging the search area. When the judging step factor is too small, the search program is controlled to start a regional search. When the step factor is too large, the line parameter can control the search program. Expand local search in local area [4]. In order to ensure the searching balance of the search program in the whole area and the local area, a balance adaptive adjustment parameter is added to make the search program perform distributed synchronous search.

The calculation formula for this step size factor is:

$$
A=l_{\max }-\frac{Q\left(l_{\max }-l_{\min }\right)}{\varepsilon Z} \times \frac{H_{1}-H_{2}}{H_{1}}
$$

In equation (1): $A$ represents the $Q$ th search step size, where $Q$ represents the current number of times; $l_{\text {max }}$ represents the maximum upper limit of the step size factor; $l_{\min }$ represents the minimum lower limit of the step size factor [5]; $\varepsilon$ represents the introduced adjustment parameter; $\mathrm{Z}$ represents the maximum total number of iterations; $H_{1}$ represents the optimal function at the initial fitness; $H_{2}$ represents the optimal function suitable for this iteration. At the same time, under the search control of this parameter, the probability of finding the approximate location of the line fault of the wind turbine is calculated. In CS technology, the discovery probability value is generally between $0.2-0.3$, and the probability under this parameter is set to $p$. When the probability is always at a large value, the convergence speed of defect search can be accelerated. When the value of the probability $\mathrm{P}$ is found to be small, the convergence speed at this time is reduced, so the optimal fitness is set by the ratio of the optimal fitness and the previous solution:

$$
F= \begin{cases}1 & f_{i}>f_{i i} \\ 0 & f_{i} \leq f_{i i}\end{cases}
$$

In equation (2): $f_{i}$ represents the optimal fitness of the previous generation;

represents the fitness of the $i$ th solution under the action of $l_{s}[6]$. According to the above calculation formula, freely adjusting the fitness of the discovery probability, the calculation result of the obtained discovery probability is as follows: 


$$
\left\{\begin{array}{l}
p^{\prime}=\sum_{i=1}^{M} u_{i} / e \\
p=p_{\min }+\left(p_{\max }-p_{\min }\right) \cdot p^{\prime}
\end{array}\right.
$$

In equation (3): ${ }^{p^{\prime}}$ represents the high-quality probability of the solution after iteration; $u_{i}$ represents the optimal fitness for the ${ }^{i}$ th solution; e represents the total number of solutions; $p_{\min }$ and $p_{\max }$ represent the upper and lower limits of the discovery probability respectively . Combine the above two sets of formulas to get a search program, implement a defect search instruction for the power system, and obtain the approximate area range of the wind turbine line fault, as shown in Figure 1 below.

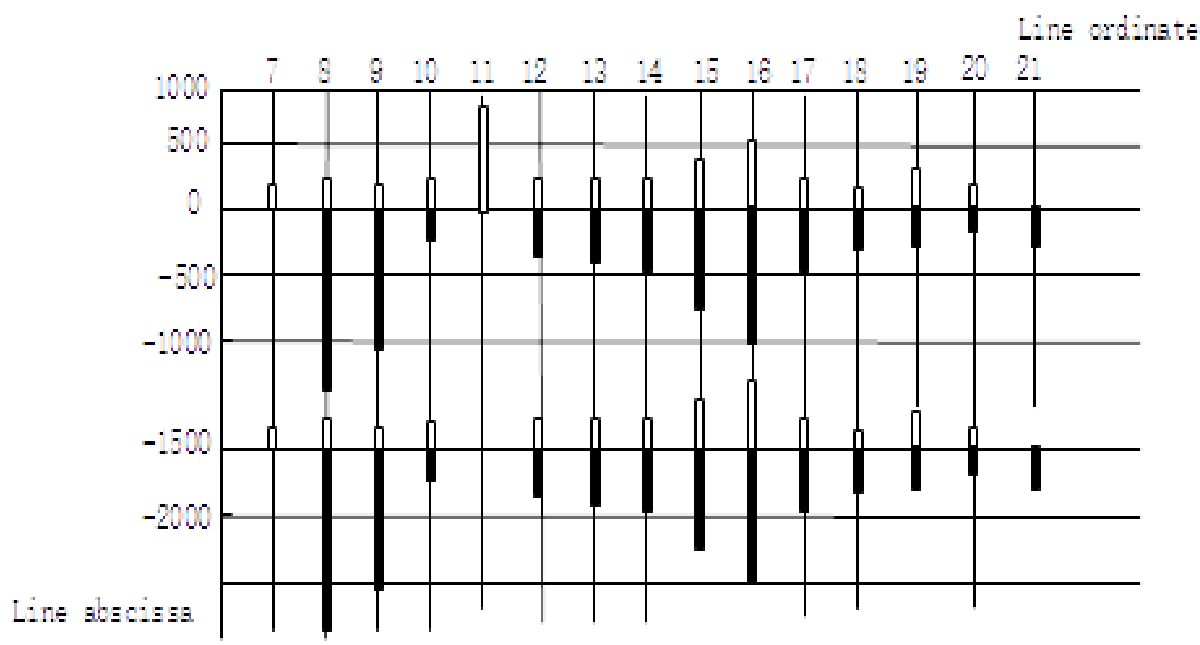

Fig. 1. Defining scope of wind turbine line faults.

The recognition result in the figure above is the target range of wind turbine line faults. However, this target range not only contains defective nodes, but also contains a small number of normal nodes. Therefore, the data of the defective nodes need to be matched and processed.

\subsection{Fault data matching processing}

After obtaining the defined fault range of the wind turbine line, the main characteristics of the original wind turbine line are detected and matched with the characteristic points of the fault of the wind turbine line to be matched. Set the characteristic point of the line of the original wind turbine as ${ }^{a_{i}}$ and the characteristic point of the line fault of the wind turbine to be matched as ${ }^{b_{i}}$, then:

$$
\left\{\begin{array}{l}
a_{i}=\left(\begin{array}{lll}
x_{i}^{\prime \prime} & y_{i}^{\prime \prime} & z_{i}^{\prime \prime}
\end{array}\right) \\
b_{i}=\left(\begin{array}{lll}
x_{i}^{\prime} & y_{i}^{\prime \prime} & z_{i}^{\prime}
\end{array}\right)
\end{array}\right.
$$


In equation (4), $i=0,1,2, \ldots n$, there are $n$ pairs of feature points in total; $w$ represents the point cloud set corresponding to $a_{i}$, and $v$ represents the point cloud set corresponding to $b_{i}$ [7].

Taking point cloud $\mathrm{W}$ as a reference, rigid transformation is performed on point cloud $\mathrm{V}$, and $\mathrm{V}$ is transformed into the coordinate system of $\mathrm{W}$, and the matrix is as follows:

$$
w_{i}=\left(\begin{array}{lll}
\alpha_{00} & \alpha_{01} & \alpha_{02} \\
\alpha_{10} & \alpha_{11} & \alpha_{12} \\
\alpha_{20} & \alpha_{21} & \alpha_{22}
\end{array}\right) v_{i}+\left(\begin{array}{l}
\beta_{x} \\
\beta_{y} \\
\beta_{z}
\end{array}\right)
$$

The definition of each variable is the same as the above formula. Due to the certain mapping relationship between the fault points in the wind turbine line and the matched threedimensional point cloud, the matching points in the three-dimensional space of the wind turbine line fault are obtained. as shown in Figure 2.

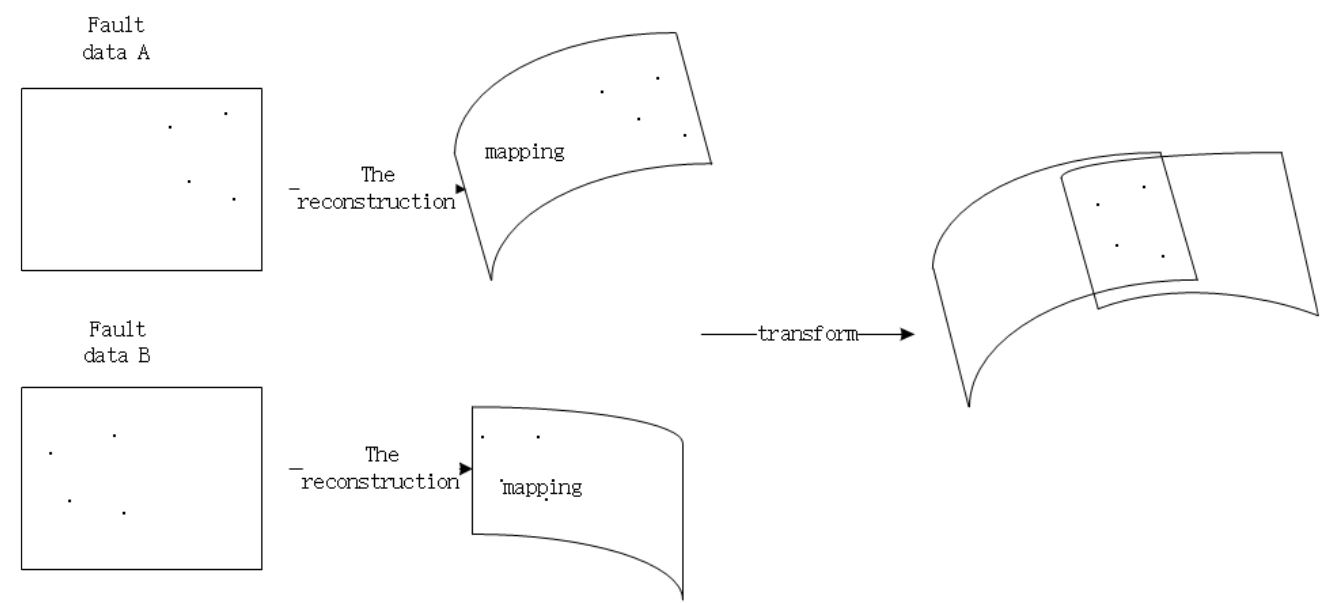

Fig. 2. Schematic diagram of line fault points matching for wind turbines

As shown in Figure 2, the characteristic points in the wind turbine line are matched with the three-dimensional spatial fault points of the wind turbine line, but because of the matching process, it may be disturbed by random factors [8]. To this end, in the neighborhood of feature points, reconstruction of inter-point interpolation is required to replace defective points with missing phases. Extend the $\mathrm{X}$ direction or $\mathrm{Y}$ direction of the position to be matched after matching. When two points are found to be defective, solve the three-dimensional space where the two points are located. Use the points obtained by interpolation to replace the original defect points and calculate. The process is as follows: 


$$
\left\{\begin{array}{l}
w_{i}^{\prime}=\frac{\left(w_{i}^{p l e}+v_{i}^{p r e}\right)}{2} \\
v_{i}^{\prime}=\frac{\left(w_{i}^{b x k}+v_{i}^{b x k}\right)}{2}
\end{array}\right.
$$

Where $\left(w_{i}^{p r \varepsilon}, w_{i}^{b x x k}\right)$ represents the point where interpolation replaces $w_{i}$, and $\left(v_{i}^{p l \varepsilon}=v_{i}^{b x k}\right)$

represents the point where interpolation replaces $v_{i}$.

The specific replacement diagram is as follows:

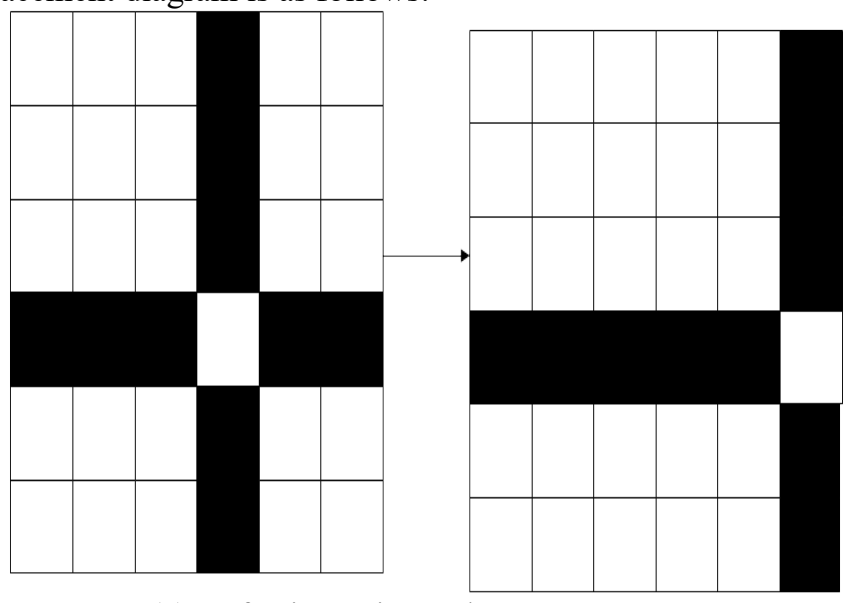

(a) Defective point replacement process 


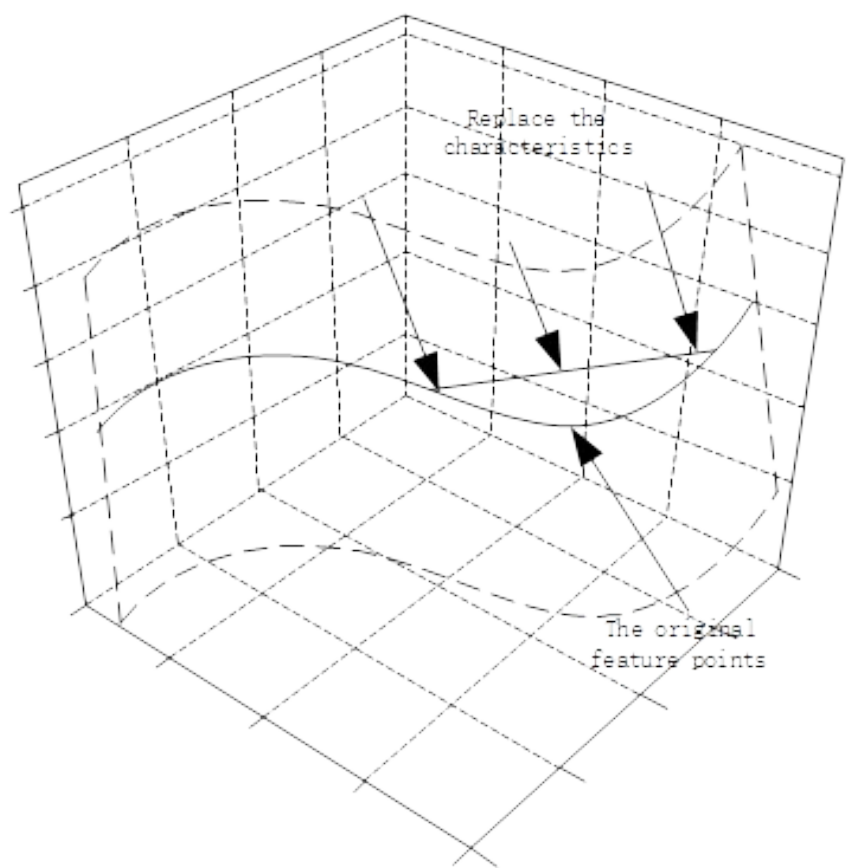

(b) Space point replacement process

Fig. 3 Schematic diagram of defect replacement process

As shown in Figure 3, the nodes in the Figure 3(a) are used to represent nodes. The filled squares indicate defects or faults at the location, the upper part indicates that the original wind turbine line contains characteristic points, and the lower part indicates the wind turbine to be matched Corresponding defect fault points in the line. Figure 3(b) shows the cross-section lines obtained after matching in the wind turbine line. So far, the fault data matching process is completed, and the fault data deviation correction is implemented on this basis.

\subsection{Error data deviation correction}

In the process of matching fault data of wind turbine units, due to the influence of random factors on the fault location of each line, a certain deviation occurs. In order to accurately locate the fault point of the line, the fault data deviation needs to be corrected. The fault data deviation is detected by using the wind turbine circuit diagram. On the edge of the wind turbine circuit fault, the mark is left blank, as shown in Figure 4:

$$
\text { 4The left tag- }
$$$$
-{ }_{\text {To the right }}
$$

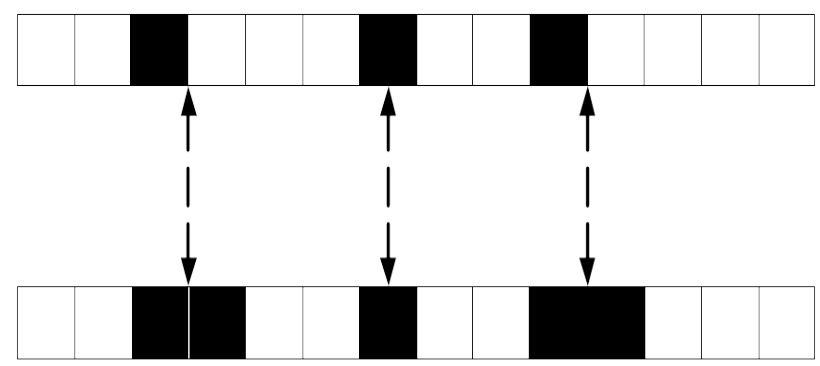


Fig. 4 Marking the fault edge of a wind turbine line

According to the actual situation, if the distance between the two color scales remains unchanged, there is no deviation between the two marks, otherwise the fault data deviation correction is implemented [9]. To this end, a fault data deviation correction model is established and the fault data deviation is revised. The specific structure of the revision process is shown in Figure 5:

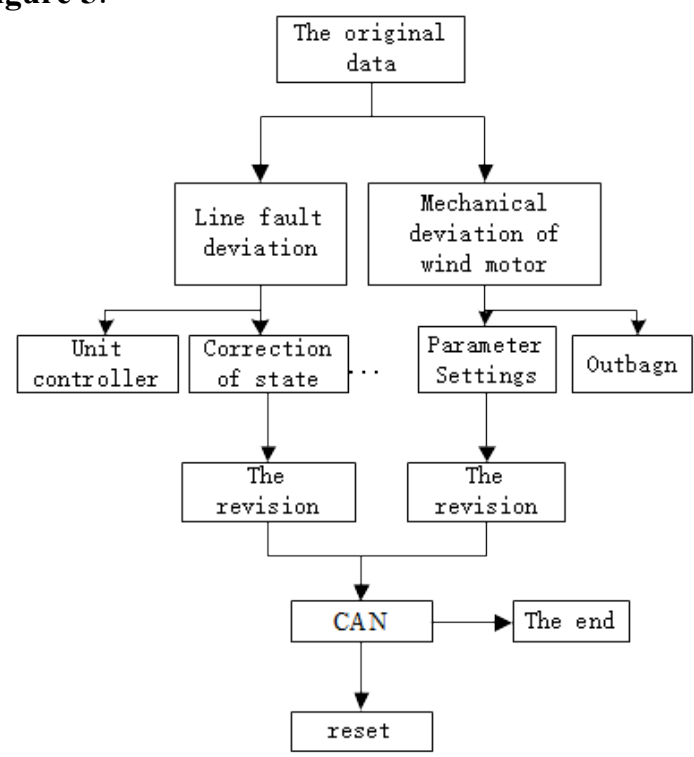

Fig. 5 Schematic diagram of error data deviation correction

As shown in Figure 5, the master-slave structure of the fault data deviation correction model is used to control the deviation generated in the wind turbine line. The control effect is divided into two parts: the mechanical deviation of the wind turbine and the deviation of the line fault. Among them, the correction of the mechanical deviation of the wind turbine must be completed by setting the relevant parameters and the correction of the state of the wind turbine equipment during operation. The line fault deviation revision uses the unit controller, photoelectric encoder, motor drive and other related equipment to modify the line fault deviation in real time, and control and display it. Both parts can complete the fault data deviation correction through the CAN bus, so that the wind turbine line fault is guaranteed. The photoelectric encoder installed on the line main shaft in the wind turbine equipment runs simultaneously with the operation of the wind turbine control unit. The specific physical diagram is shown in Figure 6. 


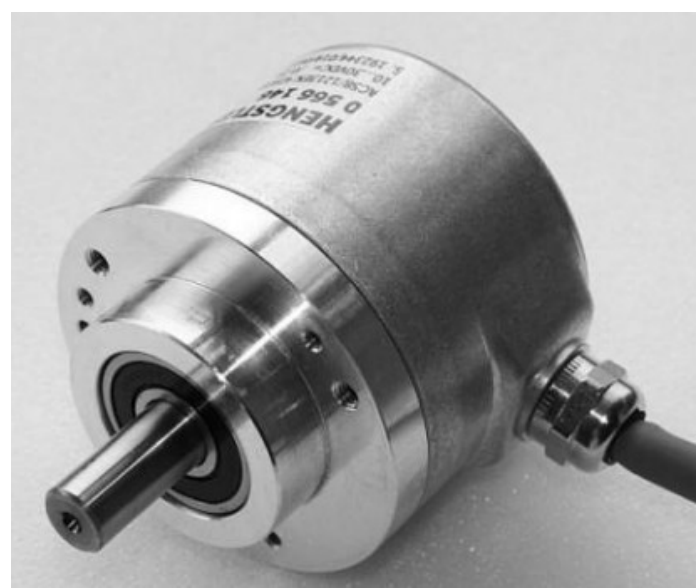

Fig. 6 Physical picture of photoelectric encoder

\subsection{Power system wind turbine line fault location}

After the fault data deviation is corrected, the fault current travelling wave head arrival time is calibrated. In the wind turbine line, the traveling wave signal is decomposed into components with different characteristic scales, and the instantaneous maximum value is obtained for the decomposed component to calibrate the wave head arrival time. When the wind turbine line fault is in the fault transient state, the current signal will generate highfrequency components due to the fault. The fault signal is decomposed by EMD, and the highest frequency component in the decomposed signal is Hilbert transformed to obtain the fault signal. Instantaneous frequency graph. Solve the maximum value of the instantaneous frequency to remove the interference wave head [10]. In the time-frequency diagram, when the fault traveling wave head appears as the first maximum value, the time when the fault traveling wave head reaches the detection point can be determined. In addition to locating the fault area of the wind turbine line, in addition to knowing when the fault traveling wave head reaches the detection point, it is also necessary to calculate the traveling wave velocity. When the frequency-dependent interference of the relevant parameters of the wind turbine line is not considered, the formula for calculating the traveling wave velocity $\mathrm{v}$ of a fault is as follows:

$$
v=\frac{1}{\sqrt{N V}}
$$

Among them, $N$ is the inductance value of the unit length wind turbine line, and $V$ is the capacitance value of the unit length wind turbine line. Theoretically, it is feasible to calculate the traveling wave velocity of a fault using a formula, but in practical applications, because the wind turbine unit line is usually constructed across regions, the inductance, capacitance, and resistance of the line will change as the line grows. The wave velocity formula is as follows.

$$
J=\frac{v}{\sqrt{\frac{1}{2}\left[\omega^{2} N-V G+\sqrt{\left(G^{2}+\omega^{2} C^{2}\right)}\right]}}
$$

In equation (8), $\mathrm{G}$ is the conductance value of the unit wind turbine unit line, and $\omega$ is the angular frequency. The figure is a schematic diagram of the wind turbine line, and the specific 
principle of measuring the traveling wave velocity of a fault is explained in detail.

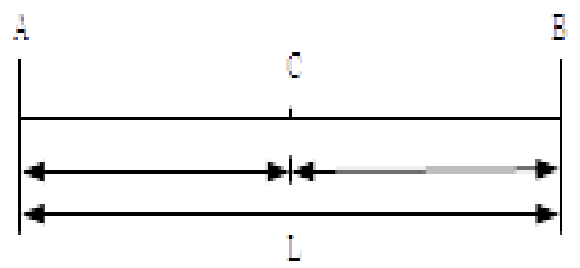

Fig. 7 Schematic diagram of the wind turbine

As shown in Figure 7, the length of the line is 1, detection points A and B are placed at both ends of the line, and detection points $C$ are set at $1 / 2$. In practical applications, the detection points are not placed at equal intervals, and the clocks of the detection points at different intervals are implemented by tracking. After a fault occurs, the fault occurrence section is judged according to the phase separation phase difference of the fault current.

Assume that the fault occurs in the $\mathrm{BC}$ section, and the time ${ }^{t_{B}}$ and ${ }^{t_{C}}$ at which the fault traveling wave reaches the detection points $\mathrm{B}$ and $\mathrm{C}$ respectively. Calculate the transmission

time ${ }^{t_{B C}}$ on the line in the BC section. Simply calculate the fault traveling wave wave speed. Simply calculate the fault traveling wave velocity, and similarly calculate the AC traveling fault wave velocity. After the line fails, the information collected by the fault information collector at each monitoring point is sent back to the monitoring center. After receiving the data, the monitoring host obtains the arrival time of the wave head from the wavelet scale transformation according to the fault identification result, analyzes the section where the fault point is based on the principle of phase-separated current and phase difference, and calculates the traveling wave velocity of the fault to locate the fault area of the motor unit circuit. At this point, the fault location of the wind turbine in the power system is completed.

\section{Experiment}

In order to verify the fault location method of the power system wind turbine line designed in this paper, experiments are designed to verify whether the location information and obstacle source are accurate in the event of multiple faults in the wind turbine line.

\subsection{Experiment preparation}

The wind power unit of the power system is used as the experimental research object, including 1 breakpoint failure point, 10 operating failure points and 5 positioning points. And experimental simulation diagram are shown in Figure 8. 


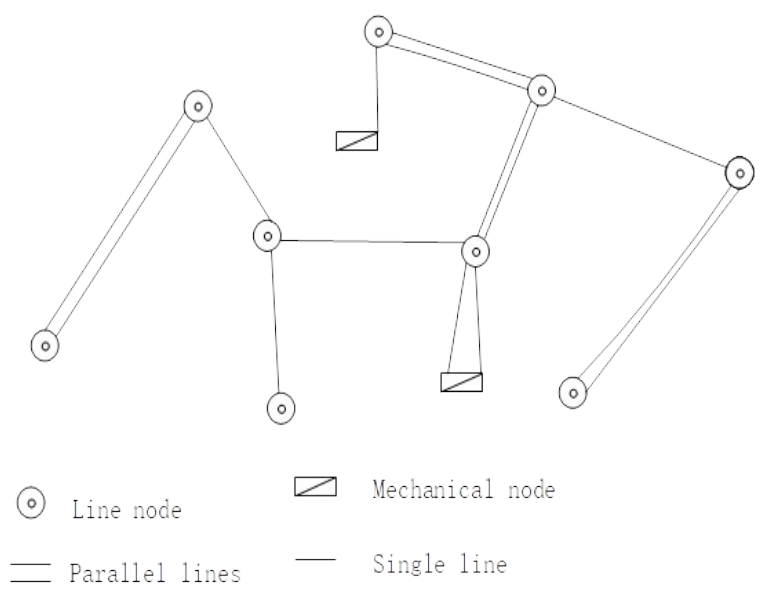

Fig. 8 Experimental simulation diagram

The coordinates of the fault point and each set anchor point are shown in the following Table 1:

Table 1. Coordinates of failure points and various set anchor points

\begin{tabular}{cccc}
\hline $\begin{array}{c}\text { Experiment } \\
\text { library number }\end{array}$ & X-axis & Y-axis & Occurrences / times \\
\hline P1 & 34 & 114 & 2 \\
P2 & 25 & 53 & 2 \\
P3 & 64 & 158 & 2 \\
P4 & 57 & 25 & 2 \\
P5 & 142 & 58 & 1 \\
P6 & 153 & 114 & 1 \\
P7 & 176 & 14 & 1 \\
P8 & 134 & 174 & 1 \\
P9 & 176 & 25 & 1 \\
P10 & 134 & 2 \\
\hline
\end{tabular}

Under the above experimental conditions, the traditional method and the method in this paper were used to locate and analyze the experimental results.

\subsection{Experimental results and analysis}

Under the above experimental conditions, two methods were used to locate the data, and the test point results were obtained. The experimental results are shown in Table 2:

Table 2 Comparison of positioning results of the two methods

\begin{tabular}{|c|c|c|c|}
\hline $\begin{array}{l}\text { Experiment } \\
\text { number }\end{array}$ & Experiment set point & $\begin{array}{l}\text { Experimental results of the } \\
\text { method of this paper }\end{array}$ & $\begin{array}{l}\text { Experimental results of } \\
\text { traditional method }\end{array}$ \\
\hline $\mathrm{P} 1$ & $1 、 2 、 9 、 10$ & $1 、 2 、 9 、 10$ & $1,9,10$ \\
\hline $\mathrm{P} 2$ & $4,5,6$ & $4,5,6$ & $4,5,6$ \\
\hline P3 & $3,7,8$ & $3,7,8$ & $3 、 4 、 8$ \\
\hline P4 & $11 、 15,16$ & $11 、 15,16$ & $11 、 13,9$ \\
\hline P5 & $8,5,10$ & $8,5,10$ & $8,5,10$ \\
\hline
\end{tabular}




\begin{tabular}{|c|c|c|c|}
\hline P6 & $15,12 、 13$ & $15,12 、 13$ & $15 、 12 、 13$ \\
\hline P7 & $6 、 7,9$ & $6 、 7 、 9$ & $6 、 5,9$ \\
\hline P8 & $14 、 2 、 4$ & $14 、 2 、 4$ & $14 、 4$ \\
\hline P9 & $8,11,9$ & $8,11,9$ & $8 、 10,9$ \\
\hline P10 & $2,6,7$ & $2 、 6 、 7$ & $2 、 5 、 7$ \\
\hline
\end{tabular}

As can be seen from Table 2, compared with the traditional method, the positioning results of this article are based on the experimentally set point coordinates. The basic positioning points of this method are correct, and the experimental results appear marked. The smaller the distance between the wind turbines, the more accurate the positioning results. Omissions and errors in the number of anchor points make the method in this paper more applicable.

\section{Conclusion}

This paper designs a fault location method for power system line faults, and solves the problem of inaccurate or missing multi-points in conventional fault location methods for power system line faults. It is hoped that the research in this paper can provide help for fault line maintenance of power units.

In future research, we will focus on improving the efficiency of power system line fault location and detection, and further improve the proposed algorithm.

\section{References}

[1]Huang, X., Zhang J., Tian, Y., et al.: On-line monitoring system for wind power busbar trunk heat fault status. Power System Protection and Control, 45(24): pp. 130-137 (2017)

[2]Yu, Z., Zhu, W.: Study on stability for delayed power systems with wind turbine generators. Control Engineering of China, 25(10): pp. 1871-1875 (2018)

[3]Qi, Y, Zhao, P., Gao, Sheng-li, et al.: A new fault monitoring and fault diagnosis strategy for wind turbine bearings 25(01): pp 37-43 (2018)

[4]Ren, Y., Zhang, K.: Integrated condition monitoring and fault diagnosis technology for wind turbine drive-train. Journal of Drainage and Irrigation Machinery Engineering, 36(07): pp 613-616 (2018)

[5]Jia, Z., Gu Y.. Fault Diagnosis of wind turbines based on improved multiblock kernel principal component analysis[J]. Journal Of Chinese Society Of Power Engineering, 38(10): pp 820-828 (2018) [6]Tan Bown, Qiu Yingning, Ui Dan, et al.: Thermal-stress analysis of rolling bearing failure at highspeed end of a wind turbine gearbox[J]. Journal Of Chinese Society Of Power Engineering, 37(02): pp 119-125 (2017).

[7]LI J, Li M, Wang H, et al.: Sparse fault diagnosis method for rolling bearings of wind turbines based on COMP algorithm[J]. China Mechanical Engineering, 29(12): pp 1428-1433 (2018).

[8]Xu Q, Bai K, Xu H, et al.: Research status and development trend of wind power gearbox condition monitoring and fault diagnosis[J]. Lubrication Engineering, 44(08): pp 138-147 (2019).

[9]Shi P, Liang K, Zhao N, et al.: Fault Diagnosis of Wind Turbine Gearbox Bearing Based on Fractal Dimension and GA-SVM[J]. Acta Metrologica Sinica, 39(01):pp 61-65 (2018).

[10]LI H, SUN D, SHEN Y.: Application of EEMD noise reduction and cepstrum analysis in fault diagnosis of wind power bearing[J]. Machine Tool\&Hydraulics, 46(13): pp 156-159(2018). 